\title{
Resolution of the Computational Diffusion Hydrodynamic Model into Partial Differential Equation Form
}

\author{
Theodore V. Hromadka II ${ }^{1}$, Colin Bloor ${ }^{2}$, Prasada Rao ${ }^{3}$ \\ ${ }^{1}$ Department of Engineering-Mathematics, United States Military Academy, West Point, USA \\ ${ }^{2}$ Hromadka \& Associates, Rancho Santa Margarita, USA \\ ${ }^{3}$ Department of Civil and Environmental Engineering, California State University, Fullerton, USA \\ Email: ted@phdphdphd.com
}

How to cite this paper: Hromadka II, T.V., Bloor, C. and Rao, P. (2018) Resolution of the Computational Diffusion Hydrodynamic Model into Partial Differential Equation Form. Journal of Water Resource and Protection, 10, 1175-1184.

https://doi.org/10.4236/jwarp.2018.1012069

Received: November 22, 2018

Accepted: December 25, 2018

Published: December 28, 2018

Copyright $\odot 2018$ by authors and Scientific Research Publishing Inc. This work is licensed under the Creative Commons Attribution International License (CC BY 4.0).

http://creativecommons.org/licenses/by/4.0/

\begin{abstract}
Computational modeling continues to evolve in applications of hydrology and hydraulics, and the field of Computational Hydrology and Hydraulics has grown into a significant technology in both engineering and computational mathematics. In this paper, the fundamental issue of assessment of computational error is addressed by determination of an "equivalent" mathematical statement, as a partial differential equation ("PDE") that describes the original mathematical PDE statement and computational solution of it. In other words, given that the computational model does not exactly solve the governing PDE and that the computational processes used to approximate the governing PDE further moves the computational outcome away from the exact solution, what "alternate" or "equivalent" PDE does the resulting computational model exactly solve? In this paper it is shown that development of such an equivalent PDE enables an assessment of computational error by direct comparison of the equivalent $\mathrm{PDE}$ to the original PDE targeted to being solved. As an example, the USGS Diffusion Hydrodynamic Model ("DHM") is examined as to development of an equivalent PDE that describes the DHM computational modeling outcome, which is then compared to the actual outcomes resulting from application of the DHM model.
\end{abstract}

\section{Keywords}

Numerical Modeling, Equivalent PDE, One and Two Dimensional Flows, Transient Simulation

\section{Introduction}

The Diffusion Hydrodynamic Model (or "DHM") is a two-dimensional flow 
routing model of the governing flow equations that describes the movement of flood flows over topographic surfaces. Originally developed for the United States Geological Survey in the early 1980s for assessment of dam break floodplain inundation, the model has been applied to numerous flood problem types including floodplain assessment, rainfall-runoff modeling, channel routing and channel/floodplain interface investigation [1]-[7]. Several other two-dimensional computer models [8] were subsequently developed after publication of the USGS Report that included the DHM computer code listing. In this work, the underpinnings of the computational algorithms used in the DHM are examined by determination of the resulting mathematical statement obtained by taking the limit of the DHM numerical statement as grid size approaches zero in the limit. That is, the typical procedure in use of such two-dimensional models is to discretize the problem domain into grids or finite volumes according to some mesh description, and then applying the governing flow equations on each grid or finite volume to arrive at a numerical statement associated with each grid or finite volume. The ensemble of these numerical statements form a matrix system that requires a solution to obtain the desired computational results of water surface elevation or flow rate or other variable of interest. Some computer codes, including the DHM, use an explicit finite difference formulation that computes the target output variable approximations at prescribed time step intervals, in order to approximate the time derivative term of the flow equations.

In this paper, the numerical statement developed by the DHM is examined as the grid size approaches zero in the limit. The limiting numerical statement is shown to be another partial differential equation ("PDE") that describes the DHM approach. That is, the original flow equations are approximated by a computational numerical statement associated with each grid of the modeling mesh discretization of the problem domain. This numerical statement includes various assumptions and simplifications to the governing flow equations. As the computational mesh dimension approaches zero in the limit, the numerical statement converges to an alternate PDE. The alternate PDE is then examined computationally and shown to describe the DHM performance, using the computer spreadsheet program EXCEL. This approach to examining numerical model convergence properties may be useful with other computational models.

\section{Flow Equations}

The theoretical basis behind flood plain hydraulics and the associated numerical models has been reviewed by Singh [9] and Hunter et al. [10]. The mathematical relationships in a one-dimensional diffusion hydrodynamic (DHM) model are based upon the continuity and momentum equations [11] as

$$
\begin{gathered}
\frac{\partial Q_{x}}{\partial x}+\frac{\partial A_{x}}{\partial t}=0 \\
\frac{\partial Q_{x}}{\partial t}+\frac{\partial\left(Q_{x}^{2} / A_{x}\right)}{\partial x}+g A_{x}\left(\frac{\partial H}{\partial x}+S_{f x}\right)=0
\end{gathered}
$$


where $Q_{x}$ is the flowrate; $x, t$ are spatial and temporal coordinates; $A_{x}$ is the flow area; $g$ is the gravitational acceleration; $H$ is the water surface evaluation; and $S_{f x}$ is a friction slope. It is assumed that $S_{f x}$ is approximated from Manning's equation for steady flow by

$$
Q_{x}=\frac{1.486}{n} A_{x} R^{2 / 3} S_{f x}^{1 / 2}
$$

where $R$ is the hydraulic radius; and $n$ is a flow-resistance coefficient which may be increased to account for other energy losses such as expansions and bend losses.

Letting $m_{x}$ be a momentum quantity defined by

$$
m_{x}=\left(\frac{\partial Q_{x}}{\partial t}+\frac{\partial\left(Q_{x}^{2} / A_{x}\right)}{\partial x}\right) / g A_{x}
$$

Equation (2) can be rewritten as

$$
S_{f x}=-\left(\frac{\partial H}{\partial x}+m_{x}\right)
$$

Rewriting Equation (3) and including equations 4 and 5, the directional flow rate $\left(Q_{x}\right)$ is computed by

$$
Q_{x}=-K_{x}\left(\frac{\partial H}{\partial x}+m_{x}\right)
$$

where $K_{x}$ is a type of conduction parameter defined by

$$
K_{x}=\frac{1.486}{n} \frac{A_{x} R^{2 / 3}}{\left|\frac{\partial H}{\partial x}+m_{x}\right|^{1 / 2}}
$$

Substituting the flow rate formulation of equation 6 into Equation (2) gives a diffusion type of relationship

$$
\frac{\partial}{\partial X} K_{X}\left[\frac{\partial H}{\partial X}+m_{X}\right]=\frac{\partial A_{X}}{\partial t}
$$

The one-dimensional model of Akan and Yen [11] assumes $m_{X}=0$ in equation 7. Thus, the one dimensional DHM flow equation is given by

$$
\frac{\partial}{\partial X} K_{X} \frac{\partial H}{\partial X}=\frac{\partial A_{X}}{\partial t}
$$

Assumptions other than $m_{X}=0$ in equation 8 result in a family of models, as summarized below.

$$
m_{x}=\left\{\begin{array}{lc}
\frac{\partial\left(Q_{x}^{2} / A_{x}\right)}{\partial X} / g A_{X} & (\text { convective acceleration model) } \\
\frac{\partial Q_{X}}{\partial t} / g A_{X} & \text { (local acceleration model) } \\
\frac{\partial Q_{X}}{\partial t}+\frac{\partial\left(Q_{x}^{2} / A_{X}\right)}{\partial X} / g A_{X} & (\text { fully dynamic model) } \\
0 & (\mathrm{DHM})
\end{array}\right.
$$




\section{Numerical Approximation}

The following steps are taken in the one dimensional model where the flow path is assumed initially discretized by equally spaced nodal points with a Manning's $\mathrm{n}$, an elevation, and an initial flow depth (usually zero) defined:

1) between nodal points along a spatial direction, compute an average Manning's $n$, and average geometric factors,

2) assuming $m_{X}=0$, estimate the nodal flow depths for the next time-step, $(t+\Delta t)$ by using Equations (7) and (9) explicitly,

3) using the flow depths at time $t$ and $(t+\Delta t)$, estimate the midtimestep value of $m_{X}$ selected from Equation (10),

4) recalculate the conductivities $K_{X}$ using the appropriate $m_{X}$ values,

5) determine the new nodal flow depths at time $(t+\Delta t)$ using Equation (19), and

6) return to Step (3) until $K_{X}$ matches midtimestep estimates.

The above algorithm steps can be used regardless of the choice of definition for $m_{X}$ from Equation (10). Additionally, the above program steps can be directly applied to a two-dimensional diffusion model with the selected $\left(m_{X}, m_{y}\right)$ relations incorporated. The two dimensional finite difference grid is shown in Figure 1.

\section{Numerical Model Formulation (Grid Element)}

For uniform grid elements, the integrated finite difference version of the nodal domain integration (NDI) method [1] is used. For grid elements, the NDI nodal equation is based on the usual nodal system shown in Figure 1. Flow rates $(q=$ $Q_{X} /$ width) across the boundary $\Gamma$ are estimated by assuming a linear trial function between nodal points.

For a square grid of width $\delta$, Equation (6) can be reduced to

$$
\left.q\right|_{\Gamma_{E}}=-\left[\left.K_{X}\right|_{\Gamma_{E}}\right]\left[H_{E}-H_{C}\right] / \delta
$$

where

$$
\left.K_{x}\right|_{\Gamma_{E}}= \begin{cases}{\left[\frac{1.486}{n} h^{5 / 3}\right]_{\Gamma_{E}} /\left|\frac{H_{E}-H_{C}}{\delta}\right|^{1 / 2} ;} & \left|H_{E}-H_{c}\right| \geq \mathcal{E} \\ 0 ; & \left|H_{E}-H_{c}\right| \geq \mathcal{E}\end{cases}
$$

In Equation (12), $h$ (depth of water) and $n$ (the Manning's coefficient) are both the average of their respective values at $\mathrm{C}$ and $\mathrm{E}$, i.e. $h=\left(h_{C}+h_{E}\right) / 2$ and $n=\left(n_{C}+n_{E}\right) / 2$. Additionally, the denominator of $K_{X}$ is checked such that $K_{X}$ is set to zero if $\left|H_{E}-H_{C}\right|$ is less than a tolerance $\varepsilon$ such as $10^{-3} \mathrm{ft}$. The net volume of water in each grid element, along the $x$ direction, between timestep $i$ and $i+1$ is

$$
\Delta q_{C}^{i}=\left.q\right|_{r_{E}}+\left.q\right|_{r_{w}}
$$

and the change of depth of water is 


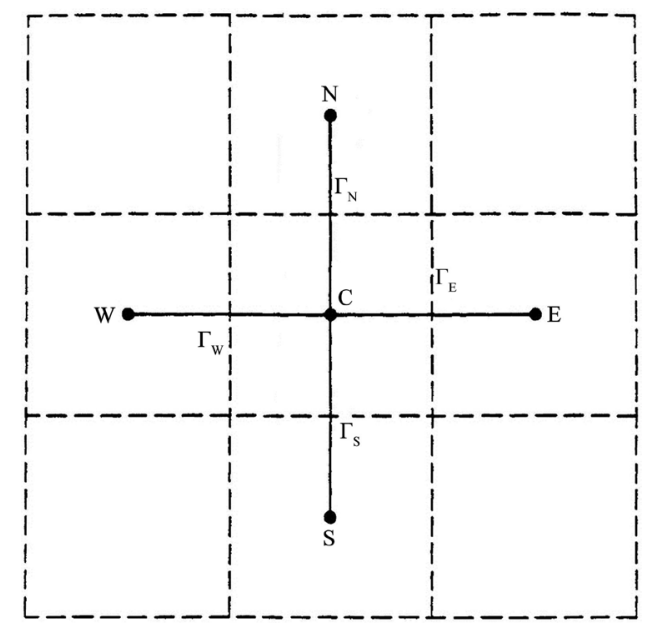

Figure 1. Two dimensional finite difference analog.

$$
\Delta H_{C}^{i}=\Delta q_{C}^{i} * \Delta t / \delta^{2}
$$

for timestep $i$ and $i+1$ with $\Delta t$ interval. Then the model advances in time by an explicit approach

$$
H_{C}^{i+1}=\Delta H_{C}^{i}+H_{C}^{i}
$$

where the assumed input flood flows are added to the specified input nodes at each timestep. After each timestep, the hydraulic conductivity parameters of Equation (12) are reevaluated, and the solution of Equation (13) reinitiated.

\section{Equivalent DHM PDE Formulation}

The mathematical statement for the DHM computational procedure, in finite difference form, as used in the DHM computer program ([1] and http://www.diffusionhydrodynamicmodel.com) was implemented using computer program EXCEL which was, in turn, applied to several test problem situations including the Example problem presented below. The alternate PDE (or, equivalent PDE) that mathematically describes the one-dimensional ("1D") formulation of the DHM is obtained by examining the limit as the spatial increments and computational time step size used in the finite difference model both approach zero in value. Upon taking the limits, the equivalent (or alternate) PDE statement is

$$
\frac{\partial}{\partial X} h^{\frac{5}{3}}\left[\frac{\partial h}{\partial X}+\alpha\right]^{1 / 2}=\frac{\partial h}{\partial t}
$$

where $\alpha$ represents the sum of relevant parameters including the gradient of the flow channel.

The above equivalent PDE is the equation that is being computationally solved, even though the original PDE was the computational goal. By comparing these two PDEs, one can see the differences and also the similarities. These variations between PDE result in describing the computational error that can be observed in using the DHM. 
It is noted that with a similar analysis, other computational models of PDE can be evaluated, and possible equivalent PDE statements derived, that describe what the computational model is actually delivering. The differences between the target PDE and the equivalent PDE provides another assessment of computational modeling error to be considered along with the other typical assessment tools contained in the modelers toolkit.

\section{EXCEL Analog of the DHM Alternate PDE}

Using the computational statement for the DHM, the modelling grid can be reduced in size, resulting, as mesh size approaches zero, in the alternate limiting numerical statement.

In order to investigate the alternate PDE form discussed above, the computer spreadsheet program, EXCEL, was used to compute a one-dimensional transient flow problem. A two-dimensional form can similarly be developed. The flow domain was established by the columns of the spreadsheet, with each column representing a single cell in the flow domain, defined by an elevation, width, and length. The time domain was established by the rows of the spreadsheet, with each row representing a single point in time, defined by the user-defined timestep. The programming code, Visual Basic, was used to solve the one-dimensional flow problem.

Flow depth was calculated at each cell based on the cell center (node), and at each timestep, by the following equation:

$$
\begin{aligned}
& \text { NewDepth }(\text { node }) \\
& =\operatorname{depth}(\text { node })+(\text { volumeFlow }(\text { node })-\text { volumeFlow }(\text { node }-1)) / \text { dimension }^{2}
\end{aligned}
$$

where $\operatorname{depth}($ node $)=$ the flow depth of the cell at the previous timestep, new Depth(node) $=$ the flow depth of the cell at the current timestep, volume Flow $($ node $)=$ the flow volume between the cell and its adjacent cell to the east, volume Flow (node-1) = the flow volume between the cell and its adjacent cell to the west, and dimension = width (which is also equivalent to length of the cell).

To calculate the flow volume between cells, the following equation was used:

$$
\text { volumeFlow }(\text { node })=\text { flowRate }(\text { node }) * \text { timestep }
$$

where the flowRate(node) was defined as:

$$
\begin{aligned}
\text { flowRate }(\text { node })= & (1.486 / \text { ManningN }) * \text { dimension } \\
& *\left(\operatorname{avgDepth}(\text { node })^{5 / 3}\right) *\left(\text { wsSlope }(\text { node })^{1 / 2}\right)
\end{aligned}
$$

where ManningN = Manning's coefficient, avgDepth = the average depth of the cell and its adjacent cell, and wsSlope(node) = the slope of the water surface elevation between adjacent cells.

\section{Example Problem}

To demonstrate the success of the alternate PDE formulation to the DHM computational statement, a one-dimensional flow problem is examined where a 
one-dimensional effectively flat domain $(0.001 \mathrm{ft}$ in $4 \mathrm{ft})$ is subjected to a nearly sudden rise in water depth at $x=0$ at model time $t=0$. The boundary condition in the EXCEL formulation is implemented by increasing the flow depth at $x=0$ over a small time interval until the target boundary condition value is achieved. A time step of 0.05 seconds, Manning's N value of 0.03 and a slope of 0.001 were used in the analysis.

The resulting computational approximation results of the alternate PDE is shown in the solid lines in Figure 2. "EXCEL \#1" illustrates the depth boundary condition vs. time in the most upstream computational element \#1. "EXCEL \#2" illustrates the resulting depth vs. time in the next downstream computational element \#2; each succeeding computational element to \#6 is similarly illustrated, showing the progression of the flow wave downstream.

To examine the equivalence of the alternate PDE to the DHM, a DHM model of the problem situation was developed and its computational results compared to the results of Figure 2. DHM model results for depths in DHM grid elements $\# 1$ to \#6 are shown as dashed lines in Figure 2. Construction of the DHM gridding was a straightforward chain of $4 \mathrm{ft} \times 4 \mathrm{ft}$ grid elements, each with a roughness coefficient of 0.03 . Slope was equivalent to $0.001 \mathrm{ft}$ drop per 4 - $\mathrm{ft}$ grid element. Grid element \#1, the most upstream, had an arbitrary elevation of 100.000 $\mathrm{ft}$. Grid element \#2 next downstream, had an elevation of $99.999 \mathrm{ft}$, and so on down to grid element \#20 which formed the outlet of the model. The outlet boundary condition was critical depth.

The only inflow boundary condition available for the DHM floodplain is a flow hydrograph. The inflow boundary condition for the EXCEL model was an implied flow hydrograph such that depth in computational element \#1 ramped up from zero at time zero to $1 \mathrm{ft}$ at 1 second, then remained constant at $1 \mathrm{ft}$ for the duration of the computation at 3 seconds. The problem then for the DHM model was to input a flow hydrograph that resulted in the depth vs. time relationship in EXCEL computational element \#1.

The inflow rate for the first 0.05 EXCEL time step was 16 cubic feet per second. While the resulting DHM depth profile approximated the EXCEL depth profile, cutting the DHM inflow at 1 second resulted in an overshoot and a depth equilibrium at $1.2 \mathrm{ft}$. Using this behavior as a baseline, the DHM inflow hydrograph was iteratively adjusted such that there was a very close match between profiles in element \#1. Eleven trials were run. The final inflow hydrograph is provided in Table 1.

The comparison of flow profiles in Figure 2 illustrates the reliability of the solution from the proposed methodology.

\section{Conclusions}

Computational models are abundant in the technical field of computational hydrology and hydraulics. The usual formulation of these models is to apply numerical methods such as Finite Element, Finite Difference, Finite Volume, 


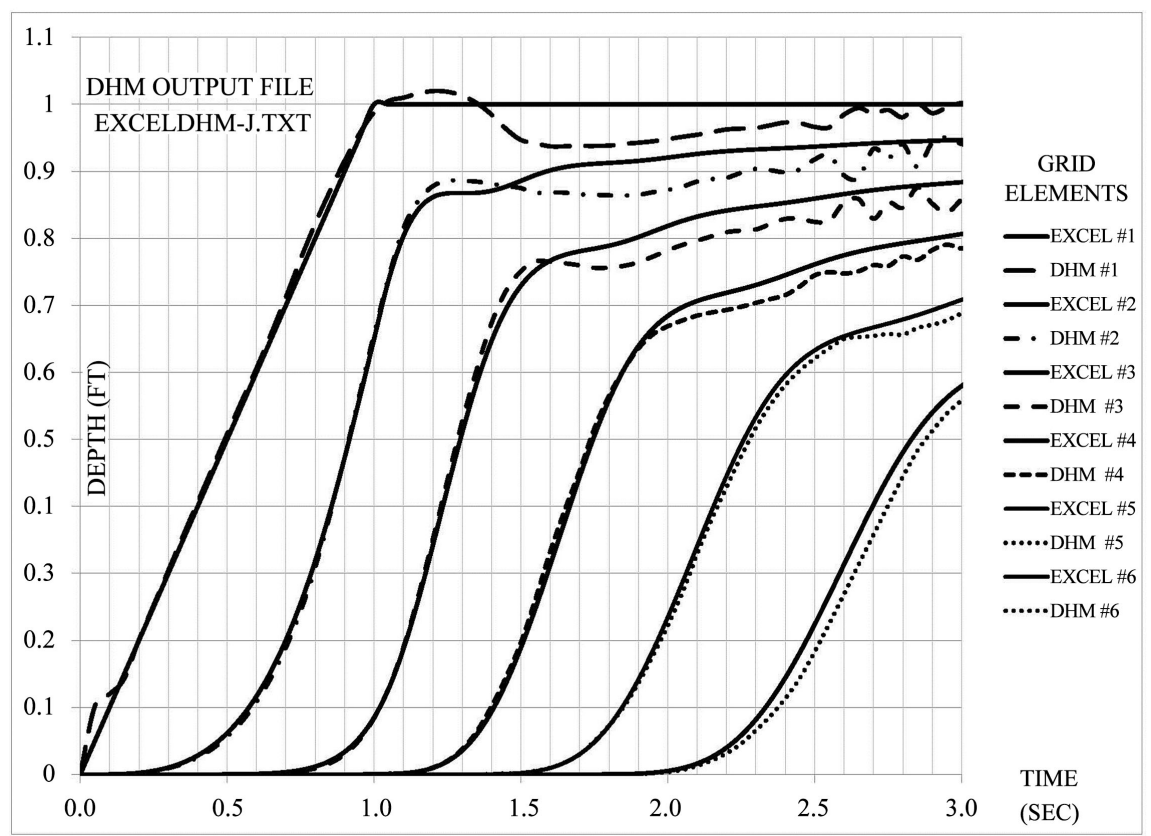

Figure 2. Comparison of transient flow depth profiles between the DHM and simplified model.

Table 1. Inflow hydrograph at Node \# 1.

\begin{tabular}{cc}
\hline Time (hours) & Flow (cfs) \\
\hline 0.0 & 16.000 \\
$2.78 \mathrm{E}-5$ & 16.160 \\
$4.17 \mathrm{E}-5$ & 16.320 \\
$5.56 \mathrm{E}-5$ & 16.640 \\
$8.33 \mathrm{E}-5$ & 17.600 \\
$1.11 \mathrm{E}-4$ & 18.880 \\
$1.67 \mathrm{E}-4$ & 24.000 \\
$2.08 \mathrm{E}-4$ & 35.000 \\
$2.64 \mathrm{E}-4$ & 50.000 \\
$2.78 \mathrm{E}-4$ & 50.000 \\
$3.19 \mathrm{E}-4$ & 40.000 \\
$4.17 \mathrm{E}-4$ & 21.357 \\
$5.69 \mathrm{E}-4$ & 25.065 \\
$6.25 \mathrm{E}-4$ & 24.529 \\
$7.50 \mathrm{E}-4$ & 22.896 \\
\hline
\end{tabular}

Boundary Element, and so forth. The selected numerical technique is used to transform the governing partial differential equation of the boundary value problem into a computational engineering mathematics ("CEM") formulation that is solved as a substitute to the analytic solution to the governing PDE. Consequently, there is a departure between the analytic solution to the PDE and BVP versus the CEM formulation. The question then becomes "What PDE does the CEM formulation solve exactly?" In other words, if the CEM formulation is an approximation of the PDE and BVP, what PDE and BVP does the CEM formulation actually solve? In the current paper, the USGS Diffusion Hydrodynamic Model ("DHM") is investigated as to determination of the "Alternate" PDE and 
BVP. Once determined, the Alternate DHM is used to demonstrate the equivalence to the CEM formulation published for the DHM and in use since the early 1970's. This Alternate DHM formulation provides significant advantages in the assessment of the performance and accuracy of DHM modeling estimates and predictions.

Resolution of the DHM computational model into its Alternate form, as accomplished in the current paper, can be done for other computational models. It is recommended that such investigation be accomplished with other computational models and the Alternate model formulation used for assessment of the modeling performance

\section{Acknowledgements}

A substantial amount of the material presented in the subject paper was authored by the paper's first author in the original USGS Report, and the relevant portions are presented only for the reader's convenience as modified for this paper. The authors wish to acknowledge the USGS and thank that agency for their permission to use this material as summarized in the current paper. The original full report and appendices are available at the subject USGS web site [1].

\section{Conflicts of Interest}

The authors declare no conflicts of interest regarding the publication of this paper.

\section{References}

[1] Hromadka, T.V. and Yen, C.-C. (1987) A Diffusion Hydrodynamic Model, U.S. Geological Survey. Water-Resources Investigations Report, USGS Publications Warehouse, 87-4137. http://pubs.er.usgs.gov/publication/wri874137

[2] Rao, P., Hromadka II, T.V., Huxley, C., Souders, D., Jordan, N., Yen, C.C., Bristow, E., Biering, C., Horton, S. and Espinosa, B. (2017) Assessment of Computer Modeling Accuracy in Floodplain Hydraulics. International Journal of Modelling and Simulation, 37, 88-95. https://doi.org/10.1080/02286203.2016.1261218

[3] Hromadka II, T.V., Berenbrokc, C.E., Freckleton, J.R. and Guymon, G.L. (1985) A Two-Dimensional Diffusion Dam-Break Model. Advances in Water Resources, 8, 7-14. https://doi.org/10.1016/0309-1708(85)90074-0

[4] Hromadka, T., Walker, T., Yen, C. and DeVries, J. (1989) Application of the USGS Diffusion Hydrodynamic Model for Urban Floodplain Analysis. JAWRA Journal of the American Water Resources Association, 25, 1063-1071. https://doi.org/10.1111/j.1752-1688.1989.tb05422.x

[5] Hromadka, T.V. and Yen, C.C. (1993) A Diffusion Hydrodynamic Model (DHM). Advances in Water Resources, 9, 118-170. https://doi.org/10.1016/0309-1708(86)90031-X

[6] Hromadka, T.V., Yen, C.C. and Bajak, P.A. (1992) Application of the USGS Diffusion Hydrodynamic Model (DHM) in Evaluation of Estuary Flow Circulation. Advances in Engineering Software, 14, 291-301.

https://doi.org/10.1016/0965-9978(92)90006-2 
[7] Hromadka II, T.V., Walker, T.R. and Yen, C.C. (1988) Using the Diffusion Hydrodynamic Model (DHM) to Evaluate Flood Plain Environmental Impacts. Environmental Software, 3, 4-11. https://doi.org/10.1016/0266-9838(88)90003-2

[8] O’Brien, J.S., Julien, P.Y. and Fullerton, W.T. (1993) Two-Dimensional Water Flood and Mudflow Simulation. Journal of Hydraulic Engineering, ASCE, 119, 244-261. https://doi.org/10.1061/(ASCE)0733-9429(1993)119:2(244)

[9] Singh, V.P. (1996) Kinematic Wave Modeling in Water Resources: Surface-Water Hydrology. Wiley, New York.

[10] Neil, H.M., Bates, P.D., Horritt, M.S. and Wilson, M.D. (2007) Simple Spatially-Distributed Models for Predicting Flood Inundation: A Review. Geomorphology, 90, 208-225. https://doi.org/10.1016/j.geomorph.2006.10.021

[11] Akan, A.O. and Yen, B.C. (1981) Diffusion Wave Flood Routing in Channel Networks. Journal of Hydraulic Division, ASCE, 107, 719-732. 